\title{
NO ADSORPTION ON ACTIVATED CARBON FIBERS FROM IRON- CONTAINING PITCH
}

Juan Alcañiz-Monge*, Agustín Bueno-López, Ma Ángeles Lillo-Rodenas, Ma José IllánGómez

Department of Inorgan ic Chemistry, Alicante University, Alicante 03080, Spain

\begin{abstract}
.
Iron-containing activated carbon fibers (Fe-ACF) have been prepared by a novel method consisting of mixing an iron precursor with raw pitch before carbon fiber formation, and further $\mathrm{CO}_{2}$ activation of the Fe-CF. The $\mathrm{NO}$ adsorption experiment revealed that iron species are accessible to gases and that they activate NO improving adsorption into the fiber microporosity. The chemisorption of NO and the subsequent dimer $\left(\mathrm{N}_{2} \mathrm{O}_{2}\right)$ formation is, in general, the rate-limiting step. Metallic iron and/or partially reduced iron compounds are the most active iron species. However, in the fibers with narrow micropores dimer diffusion in the microporosity is the slowest step and, consequently, an appropriate pore size is required from a kinetic point of view. The most suitable samples prepared were those with pores wider than $0.7 \mathrm{~nm}$ which contain partially reduced iron species. Finally, NO adsorbed can be further recovered by heating the spent adsorbents at a quite low temperature $(500 \mathrm{~K})$. Fresh and regenerated adsorbents show similar NO ad sorption capacity.
\end{abstract}

* Corresponding author: Jalcaniz@ua.es 


\section{1.- INTRODUCTION}

Activated carbon fibers (ACF) are promising solid adsorbents since they present very interesting performance in comparison to conventional activated carbons (AC). The porous texture of ACF has been object of great attention. These materials are essentially microporous $[1,2]$, being very suitable for gas-pollution control applications $[3,4]$. AC materials are receiving special attention due to their unique properties for adsorption of atmospheric pollutants, such as nitrogen oxides $\left(\mathrm{NO}_{\mathrm{x}}\right)$. Different $\mathrm{NO}_{\mathrm{x}}$ abatement processes have been proposed, AC being useful either as catalyst support or as solid reactant for $\mathrm{NO}_{\mathrm{x}}$ reduction to $\mathrm{N}_{2}$, avoiding reductant slip [5-7]. ACF can be also used in the former process but not in the latter due to the high price of ACF manufacturing, since carbon is consumed in the process. In addition, reversible NO adsorption on microporous carbon materials has been proposed. This approach presents the advantage of NO recovery, which is further used in the synthesis of valuable products. Several studies have been reported dealing with ACF utilization for this application, ACF presenting the best performance among the different porous materials studied due to their unique microporous network [8-10]. However, NO is a supercritical gas under ambient conditions, and low NO adsorption values are reached. It has been reported that NO adsorption is improved by chemisorption on suitable metals, with iron oxides showing a high activity [11]. Different iron-impregnated ACF synthesis methods, involving several steps, were checked by Kaneko et al. [12-14]. With these methods a very promising $\mathrm{NO}$ adsorption capacity $(320 \mathrm{mgNO} / \mathrm{gC}$ at $80 \mathrm{kPa}$ and $303 \mathrm{~K})$, under highly concentrated NO streams, were achieved. 
NO removal at room temperature, and from low NO concentration, is desirable in many applications, such as, diluted NO removal in highway tunnels or in enclose parking areas. In this case, high NO adsorption capacity is necessary along with rapid adsorption kinetics. On the other hand, simple manufacturing procedures are desirable for the adsorbent preparation.

In a recent article [15], the preparation of iron-containing $\mathrm{ACF}$ (Fe-ACF) was studied. An iron precursor was added to the raw pitch selected for Fe-CF preparation. 0.1 wt. \% of iron was observed to enhance drastically the activation by $\mathrm{CO}_{2}$ of the FeCF samples, also affecting the porosity of the resulting Fe-ACF.

In the current study, Fe-ACFs have been studied for NO adsorption at room temperature, analyzing the activity of the different iron species formed upon the synthesis. Iron exposure to the gas is crucial for this application and it is evaluated, since the fraction of iron located in the carbon bulk is not expected to participate in NO adsorption. The effect of the textural properties of the Fe-ACF on NO adsorption was discussed and, finally, the adsorption kinetics have been analyzed.

\section{2.- EXPERIMENTAL.}

The preparation procedure and characterization results of the Fe-ACF used in this study were described in detail elsewhere [15]. In brief, $\mathrm{FeCl}_{3}$ was carefully mixed with petroleum pitch $\left(0.001 \mathrm{gFeCl}_{3} / \mathrm{g}_{\text {pitch }}\right)$. The iron-containing pitch was spun, and the resulting Fe-CF was stabilized in air at $573 \mathrm{~K}$, carbonized in $\mathrm{N}_{2}$ at $1173 \mathrm{~K}$, and finally 
activated under $\mathrm{CO}_{2}$ flow at $1073 \mathrm{~K}$. The $\mathrm{Fe}-\mathrm{ACF}$ are denoted by $\mathrm{CFe} \%$, \% indicating the burn-off in weight percent after the activation step (for example, activated $\mathrm{Fe}-\mathrm{CF}$ with $8 \%$ burn-off is named $\mathrm{CFe} 8$ )

Table 1 summarizes some of the Fe-ACF characterization results, including the pore volumes as determined by $\mathrm{CO}_{2}(\mathrm{Vc})$ and $\mathrm{N}_{2}(\mathrm{Vn})$ adsorption at 273 and $77 \mathrm{~K}$, respectively, using the DR equation, and the different iron species identified by XRD. $\mathrm{CO}_{2}$ and $\mathrm{N}_{2}$ adsorption measurements were carried out in an automatic Quantachrome volumetric system (Autosorb-6), and X-ray diffraction patterns were recorded in a 2002 Seifert powder diffractometer. The different pore volumes used in our discussion have been calculated as follows:

- The volume of the narrowest micropores (pore size $<0.7 \mathrm{~nm}$ ), also called ultramicropores, Vc, was estimated from the $\mathrm{CO}_{2}$ DRs plots (relative pressures $<$ $0.015)$.

- The volume of the total micropores (pore size $<2 \mathrm{~nm}$ ), Vn, was calculated from the $\mathrm{N}_{2}$ DRs plots (relative pressures $<0.14$ ).

- The volume of supermicropores $(0.7 \mathrm{~nm}<$ pore size $<2 \mathrm{~nm})$ is given by the difference $\mathrm{Vn}-\mathrm{Vc}$.

NO adsorption on Fe-ACF was studied in a Stanton Redcroft thermobalance (STA-780), with $\pm 10 \mu \mathrm{g}$ accuracy. $20 \mathrm{mg}$ of sample was used for each run. The sample was treated at $373 \mathrm{~K}$ under $\mathrm{He}$ and, after weight stabilization, the temperature was decreased and maintained at the adsorption temperature $(300 \mathrm{~K}) .60 \mathrm{ml} / \mathrm{min}$ of $5 \% \mathrm{NO}$ and He balance was used in the adsorption step, which was conducted for 4 hours. The 
weight increase during adsorption was corrected with that measured in a blank experiment. After the adsorption step, the $\mathrm{NO} / \mathrm{He}$ mixture was replaced by $\mathrm{He}$, and the temperature was raised at $10 \mathrm{~K} / \mathrm{min}$ up to $473 \mathrm{~K}$ (regeneration) or $1223 \mathrm{~K}$ (heat treatment). NO was removed during these treatments and the NO adsorption capacity of the Fe-ACF samples (either regenerated at $473 \mathrm{~K}$ or heat-treated at $1223 \mathrm{~K}$ ) was further determined under similar adsorption conditions to those used for the first adsorption step $(300 \mathrm{~K} ; 60 \mathrm{ml} / \mathrm{min}$ of $5 \% \mathrm{NO} / \mathrm{He})$. The nomenclature of the samples includes the term " $r$ " and " $\mathrm{t}$ " to indicate the "regenerated" and "heat-treated samples", respectively.

In addition, the process consisted of $\mathrm{NO}$ adsorption at $300 \mathrm{~K}$ and posterior thermally treatment at $1223 \mathrm{~K}$ was also carried out with selected samples in a fixed-bed reactor coupled to a chemiluminiscence $\mathrm{NO}_{\mathrm{x}}$ analyser (Thermo Environmental Model 42H) and a gas chromatograph (Hewlett Packard, Model 5892A) for gas decomposition monitoring.

\section{3.- RESULTS AND DISCUSSION.}

\subsection{NO adsorption on Fe-ACF.}

\subsubsection{NO adsorption capacity.}

Figure 1 compiles the NO adsorption curves obtained at $300 \mathrm{~K}$ with $\mathrm{Fe}-\mathrm{ACF}$ under $5 \% \mathrm{NO} / \mathrm{He}$. In agreement with the well-known effect of $\mathrm{Fe}$, improving $\mathrm{NO}$ adsorption [12-14], all the Fe-ACF tested adsorbed NO, while similar Fe-free ACF [16] 
showed much lower NO adsorption capacity. This improvement in NO adsorption capacity on Fe-ACF with regard to Fe-free ACF indicates that, at least, is accessible to the gas flow and, as consequence, it is effective for NO adsorption.

As it is observed in the Figure 1, the non-activated fiber ( $\mathrm{CFe}$ ) showed the lowest NO adsorption capacity and, among the Fe-ACF, the sample CFe8 reached the highest NO uptake, and the higher the activation of the fiber, the lower the NO adsorption. NO adsorption on Fe-ACF could potentially depend, in one hand, on the pore volume and pore size distribution of the fiber and, on the other hand, on the nature of the iron species.

The pore volume is expected to be important for NO adsorption since NO is adsorbed in the pores of the fibers and, considering the experimental conditions used $(300 \mathrm{~K}$ and $5 \% \mathrm{NO} / \mathrm{He})$, micropores are most probably required. In Table 1 , the pore volume of samples is compiled, Vc giving information about the narrowest porosity (pore size $<0.7 \mathrm{~nm}$ ) while $\mathrm{Vn}$ takes into account the entire volume of micropores (pore size $<2 \mathrm{~nm}$ ) [17]. As observed in Table 1, CFe presents the narrowest microporosity, since both Vc and Vn values are similar. The pore volume of samples CFe8 and CFe25 is higher than that of $\mathrm{CFe}$, and samples with higher burn-off showed lower narrow micropore volume but higher supermicropore volumes $(\mathrm{Vn}-\mathrm{Vc})$. Considering these results, NO adsorption (see Figure 1) seems to depend on the narrowest microporosity. 
However, the nature of the iron species present on each sample must be also taken into account. The iron species detected by XRD on the different samples are summarized in Table 1. Note that the higher the fiber burn-off, the more oxidized the iron species are. $\mathrm{Fe}_{3} \mathrm{C}$ and different $\mathrm{Fe} / \mathrm{C}$ phases were detected on the sample $\mathrm{CFe}$ and, as the fibers were activated, the $\mathrm{Fe} / \mathrm{C}$ phases disappeared and $\alpha$-Fe was generated, which was oxidized to $\mathrm{FeO}, \mathrm{Fe}_{3} \mathrm{O}_{4}$ and $\mathrm{Fe}_{2} \mathrm{O}_{3}$ as the burn-off percentage increases. The nature of the iron species present on the Fe-ACF samples seems to be a key factor for NO adsorption. As an example, the pore volumes of $\mathrm{CFe} 25$ are higher than volumes of CFe8, while CFe8 reached the highest NO adsorption capacity, which could be related to the presence of very active reduced iron species.

\subsubsection{NO adsorption kinetics.}

The NO adsorption kinetic has been analyzed. As it is observed in the Figure 1, the shape of the curves of most samples is similar, with the only exception of the sample CFe that follows different kinetic behavior. In gen eral, NO adsorption rate is slow and, in some samples, more than 4 hours are required in order to saturate completely the adsorbent. Similar results were observed by Teng and Suuberg [18], who reported that several days were necessary for total saturation of AC adsorbents with NO. As deduced by comparing the profiles from Figure 1 with the data in Table 1, the wider the fiber porosity, the faster the adsorption rate $(\mathrm{CFe}<\mathrm{CFe} 8<\mathrm{CFe} 25)$. The low $\mathrm{NO}$ adsorption rate on Fe-ACF samples could be indicative of a kinetic barrier. This barrier is not expected to be caused by the NO molecular size, since molecules with comparable size like $\mathrm{N}_{2}$ and $\mathrm{CO}_{2}$ are able to penetrate properly into the microporous network of fibers. 
The NO adsorption rate on Fe-ACF depends on the rate of the different steps involved on the mechanism, and both NO chemisorption on iron species $\left(\mathrm{r}_{\text {chem }}\right)$ and NO diffusion throughout the microporous network of the fiber $\left(\mathrm{r}_{\mathrm{dif}}\right)$ must be considered. As all physico-chemical processes, the rate of the NO adsorption process (r) can be written as $1 / r=1 / r_{\text {chem }}+1 / r_{\text {dif. }}$ Considering that NO adsorption experiments were carried out at $300 \mathrm{~K}, \mathrm{r}_{\text {chem }}$ is expected to be slow for all the samples and, as a general trend, $\mathrm{r}_{\text {chem }}<<$ $r_{\text {dif }}$, with the exception of the sample CFe where diffusion is expected to be also slow due to the very narrow microporosity.

Different kinetic models for NO adsorption have been considered and the correspond ing mathematical expressions have been applied to the experimental profiles included in Figure 1. The resulting curves are plotted in Figure 2. Figures $2 \mathrm{a}$ and $2 \mathrm{~b}$ compile the profiles obtained assuming that the NO adsorption rate is controlled by NO diffusion throughout the microporous network of the solid, whose rate is described by the analytical solution for Fick's Law:

$$
\mathrm{m} / \mathrm{m}_{\mathrm{e}}=1-\sum_{n=0}^{n=\infty} \mathrm{a}_{\mathrm{n}} \exp \left(-\mathrm{b}_{\mathrm{n}} \mathrm{t} \mathrm{D} / \mathrm{r}^{2}\right)
$$

where $\mathrm{m}$ and me indicates the quantities adsorbed at time $\mathrm{t}$ and $\mathrm{t}=\infty, \mathrm{a}_{\mathrm{n}}$ and $\mathrm{b}_{\mathrm{n}}$ are parametres determined by the integers $n, D$ is the diffusion coefficient, $r$ the length path.

This general expression can be solved for a short period of time, obtaining the equation

$$
\mathrm{m} / \mathrm{m}_{\mathrm{e}}=\mathrm{k}_{\mathrm{s}}\left(\mathrm{t} \mathrm{D} / \mathrm{r}^{2}\right)^{1 / 2}
$$

or for a long period of time, obtaining the Lan gmuir-type equation 


$$
\mathrm{m} / \mathrm{m}_{\mathrm{e}}=1-\mathrm{k}_{\mathrm{f}} \exp \left(-\alpha_{\mathrm{f}} \mathrm{t} \mathrm{D} / \mathrm{r}^{2}\right)
$$

where the parametres $\mathrm{k}_{\mathrm{s}}, \mathrm{k}_{\mathrm{f}}$ and $\alpha_{\mathrm{f}}$ depend on the geometry of the particles.

The resulting curves obtained by equations (2) and (3) are plotted in the Figures 2a and $2 \mathrm{~b}$, respectively. Linear trends are neither observed in Figure 2a nor Figure $2 \mathrm{~b}$, indicating that NO diffusion in the fiber micropores does not determine the adsorption rate.

NO chemisorption rate can be described by the Equation of Elovich [20]:

$$
\mathrm{m} / \mathrm{m}_{\mathrm{e}}=\mathrm{a}+(1 / \mathrm{b}) \ln \left(\mathrm{t}+\mathrm{t}_{0}\right)
$$

where $\mathrm{a}$ and $\mathrm{b}$ are temperature-dependent constan ts.

The profiles obtained with equation (4) are plotted in Figure 2c, where linear trends are observed after an early period of time. Pure adsorption mechanisms are not expected to be described by Equation of Elovich [21], and therefore, it can be concluded that $\mathrm{NO}$ adsorption on Fe-ACF is controlled by the rate of NO chemisorption. As suggested by Porter and Tompkins [22], this behavior is motivated by heterogeneous surfaces or by activation energy modification as a function of surface covering. NO chemisorption on carbon or on surface oxygen-carbon complexes can be ruled out since a low adsorption temperature has been used (300 K) [23], and therefore, NO must be chemisorbed on iron species, as indicated by Kaneko [12].

The slope of the curves in Figure 2c is related to the geometry of the solid porous network. Values about 0.4 were obtained for all the activated samples, while 
0.55 was obtained for CFe. Values around 0.4 are obtained for diffusion in parallel paths [21], which is consistent to the microporous structure of ACF [24, 25]. Values around 0.55 are expected for accessible surfaces, where the adsorbed molecules can easily move without diffusional impediments, either steric or due to electrostatic interaction with the pore walls [26]. The NO adsorption on Fe-ACF samples takes place in two steps: (i) $\mathrm{NO}$ chemisorption and formation of the dimer $\mathrm{N}_{2} \mathrm{O}_{2}$, and (ii) $\mathrm{N}_{2} \mathrm{O}_{2}$ diffusion into micropores. The former step is the slowest one, and therefore, is the ratelimiting step of the NO adsorption process in Fe-ACF samples. However, dimer diffusion throughout very narrow micropores is difficult, and even slower than NO chemisorption and dimer formation, and th is has to be the case for the CFe sample.

\subsection{Spent adsorbent after-treatment.}

After NO adsorption at $300 \mathrm{~K}$, the spent adsorbents were treated at 473 and 1223 $\mathrm{K}$ under He. These heat-treatments were carried out both in a thermobalance and in a fixed-bed reactor coupled to a gas chromatograph and a specific $\mathrm{NO}_{\mathrm{x}}$ analyzer for gas composition monitoring was used. In Figure 3, the thermogravimetry profiles during heat- treatment at $1273 \mathrm{~K}$ are included, where two regions can be distinguished: below $500 \mathrm{~K}$, NO desorption takes place and the corresponding weight loss is observed; between 500 and $1200 \mathrm{~K}$, the decomposition of surface oxygen-carbon complexes (yielding $\mathrm{CO}$ and/or $\mathrm{CO}_{2}$ ), and $\mathrm{H}_{2}$ evolution is detected above $1100 \mathrm{~K}$. It is worth to mention that the thermogravimetry profiles obtained with fresh samples (without previous NO adsorption) are similar to those obtained with spent adsorbents, yielding 
$\mathrm{CO}, \mathrm{CO}_{2}$, and $\mathrm{H}_{2}$ in the described ranges of temperature, with the only exception of the absence of NO desorption below $500 \mathrm{~K}$, as it is obvious.

Both the NO desorption below $500 \mathrm{~K}$ and the processes occurring at higher temperature are described in detail in the coming sub-sections.

\subsubsection{NO desorption.}

As mentioned, $\mathrm{NO}$ adsorbed at $300 \mathrm{~K}$ on $\mathrm{Fe}-\mathrm{ACF}$ adsorbents can be further desorbed by heat-treatment of the spent adsorbents at temperatures around $500 \mathrm{~K}$, and NO desorption occurs without simultaneous emission of $\mathrm{N}_{2}, \mathrm{NO}_{2}, \mathrm{CO}$ or $\mathrm{CO}_{2}$, as it has been observed by gas chromatograph and chemiluminiscence $\mathrm{NO}_{\mathrm{x}}$ analyser. The amounts of NO adsorbed and further desorbed are similar (whatever the sample considered) indicating that the adsorption-desorption process is reversible. Several authors have reported that, in addition to reversible NO adsorption, either NO reduction by carbon (producing $\mathrm{N}_{2}, \mathrm{~N}_{2} \mathrm{O}, \mathrm{CO}$ and/or $\mathrm{CO}_{2}$ ) $[8,12,27,28]$ or $\mathrm{NO}$ disproportion into $\mathrm{N}_{2} \mathrm{O}$ and $\mathrm{NO}_{2}$ [29-31] could potentially occur, but these reactions are not taking place under our experimental conditions. Differences between the behavior of the Fe-ACF tested in the current study and the behavior described by other authors [8, 12, 27-31] must be attributed to differences in the preparation procedure of the samples. The preparation methods described in the literature consisted of ACF oxidation, further impregnation of the oxidized ACF with an iron precursor and, finally, heat-treatment at low temperature (lower than $500 \mathrm{~K}$ ), obtaining samples with high surface covering of oxygen-carbon complexes [32]. Surface oxygen-carbon complexes decomposition 
occurs along with NO desorption during regeneration, and the nascent sites created after $\mathrm{CO}$ and/or $\mathrm{CO}_{2}$ decomposition have been described as carbon atoms with unsaturated valences which are highly reactive for NO chemisorption and further reduction. On the contrary, the samples prepared in the current study by $\mathrm{CO}_{2}$ activation of $\mathrm{Fe}-\mathrm{CF}$ do not contain a significant surface covering by oxygen-carbon complexes and the stability of the existing complexes is high, they only decompose at temperatures well above $500 \mathrm{~K}$ [33]. For this reason, the Fe-ACF samples tested in the current study show negligible reactivity with NO within the regeneration range of temperature, and gaseous products of the NO-C reaction are not detected. In addition, NO could dissociate on iron sites and further react with the carbon matrix. The iron loading on the Fe-ACF samples tested (about 0.1 wt.\%) is much lower than that introduced by other authors (4-12\%), and therefore, NO dissociation on iron species is expected to hardly occur.

As observed in Figure 3, NO is rapidly desorbed from $\mathrm{CFe}$ 8, $\mathrm{CFe} 25$, and $\mathrm{CFe} 38$ after raising the temperature, while $\mathrm{CFe}$ needs higher temperatures in order to release the adsorbed NO. Among the activated samples, CFe25 shows NO desorption at the lowest temperature and $\mathrm{CFe} 8$ and $\mathrm{CFe} 38$ desorbed $\mathrm{NO}$ at slightly a higher temperature. The rapid desorption observed in activated samples rules out gas diffusion limitations in the microporous network, indicating that $\mathrm{NO}$ adsorption rate is controlled by the rate of $\mathrm{N}_{2} \mathrm{O}_{2}$ formation. This behavior has been also reported by other authors $[10,12,32]$, and samples with significant hysteres is in the NO adsorption-desorption cycle have been described. However, it has been suggested that NO chemisorbed on iron sites located at the entrance of micropores blocks this entrance hindering the desorption of NO located inside the pore. In the Fe-ACF prepared in the current study, this argument is not 
expected to be valid due to the low concentration of iron and the nature of the iron species $\left(\mathrm{Fe} / \mathrm{C}\right.$ or cementite [15]). In addition, $\mathrm{N}_{2}$ emission did not occur confirming that the amount of NO chemisorbed is negligible. It is reasonable to assume that, in our case, $\mathrm{N}_{2} \mathrm{O}_{2}$ diffusion is slow due to the about similar molecular size and pore diameter, and the high interaction en ergy $[9,12]$. The driving force for dimer dissociation is the NO concentration gradient inside and outside of the pore. This gradient does not provide enough energy for dimer dissociation and additional energy, enthalpy, is necessary $\left(\Delta \mathrm{H}_{\mathrm{dis}}\right)$. This en thalpy has been calculated from the TG profile of CFe (Figure 3) by applying the method of Newkirk [34] to the range of temperatures $480-573 \mathrm{~K}$. For a series of temperature, $T_{1}<T_{2}<T_{3}$, the remaining weight $m$ and the rate of weight loss $(d m / d t)$, were obtained by the tangents to the curves at these temperatures. For firstorder reactions the rate of weight loss is related to the remainder weight by a rate constant $k: d m / d t=k \mathrm{~m}$. Hence, we obtain different $\mathrm{k}$ values for different temperatures. Taking into account that the rate constant is related to temperature by an Arrhenius equation $k=k o \exp \left(-\Delta \mathrm{H}_{\mathrm{dis}} / R T\right)$, the enthalpy of dissociation $\Delta \mathrm{H}_{\mathrm{dis}}$ can be found from the slope of a plot of $\ln \mathrm{k}$ versus $1 / \mathrm{T}$. $\Delta \mathrm{H}_{\mathrm{dis}}$ was estimated to be $12 \mathrm{~kJ} / \mathrm{mol}$, being in good agreement with values reported by other authors $(8-16 \mathrm{~kJ} / \mathrm{mol})[8,9,12,30]$. This supports the hypothesis that the slow NO desorption from $\mathrm{CFe}$ is due to dimer diffusion limitation.

\subsubsection{Heat-treatment of samples.}

Considering that the sample $\mathrm{CFe} 8$ presented the highest $\mathrm{NO}$ adsorption capacity among the different samples studied and that its porosity is quite similar to that of 
$\mathrm{CFe} 25$, it is reasonable to expect the presence of highly active iron species on CFe8. In a previous study, the nature of the different iron species on Fe-ACF samples was analyzed, concluding that the oxidation state of iron increases with the fiber burn-off [15]. The iron species detected in the sample $\mathrm{CFe} 8$ were metallic $\mathrm{Fe}$ and $\mathrm{FeO}$. In order to analyze the potential effect of iron oxidation state in NO adsorption, the samples were treated at $1223 \mathrm{~K}$ under inert gas in order to reduce the oxidized iron species by the carbon.

Heat-treatment was analyzed by TG and gas chromatography. Figure 3 showing the thermogravimetry profiles. As indicated above, $\mathrm{CO}$ and $\mathrm{CO}_{2}$ are evolved between 500 and $1100 \mathrm{~K}$, and $\mathrm{H}_{2}$ above $1100 \mathrm{~K}$. Note that samples $\mathrm{CFe} 8$ and $\mathrm{CFe} 25$ yield high amounts of $\mathrm{CO}$ and $\mathrm{CO}_{2}$, which are much higher than those emitted by $\mathrm{CFe} 38$. Sample CFe emitted little $\mathrm{CO}$ and $\mathrm{CO}_{2} . \mathrm{H}_{2}$ production is about similar in all the samples.

$\mathrm{CO}$ formation due to the consumption of carbon in the reduction of the iron species [35] is expected to be negligible since the iron loading is very low (around 0.1 wt. \%). Therefore, $\mathrm{CO}$ and $\mathrm{CO}_{2}$ emission has to be a consequence of decomposition of surface oxygen-carbon complexes, which were formed during the $\mathrm{CO}_{2}$ activation step. For this reason, the sample $\mathrm{CFe}$ did not emit $\mathrm{CO}$ and $\mathrm{CO}_{2}$ while the samples $\mathrm{CFe} 8$ and CFe25 present a high surface coverage of oxygen complexes. Sample CFe38, which reaches the highest activation burn-off, has a quantity of surface oxygen complexes smaller than on $\mathrm{CFe} 8$ and $\mathrm{CFe} 25$, suggesting that an external fiber consumption mainly occurred. 
$\mathrm{H}_{2}$ emission above $1100 \mathrm{~K}$ is related to the preparation procedure of the samples, including carbonization at $1173 \mathrm{~K}$ and further activation at a lower temperature (1073 K) [15]. Since heat-treatment was extended to a temperature higher than that of carbonization, $\mathrm{H}_{2}$ emission takes place once the temperature of the previous carbonization is almost exceeded.

\subsection{NO adsorption on regenerated and heat-treated samples.}

\subsubsection{NO adsorption on regenerated samples.}

Sample regeneration is important both from a technical and economical point of view. NO is a valuable gas that can be used for synthesis of different chemicals. Adsorbent regeneration must be fast and the process must take place at low temperature. On the other hand, NO must be recovered without emission of additional gasses, such as those generated by NO disproportion and/or NO-carbon reaction. As described in the previous section, the Fe-ACF tested in this study accomplishes all those requirements, because, as NO adsorbed on Fe-ACF can be further recovered by heat-treatment at around $500 \mathrm{~K}$. For this process, we chose a slightly lower temperature, $473 \mathrm{~K}$, for which almost all NO has been removed, to ensure that only NO is evolved (according to the previous TG measurements).

Adsorbent reutilization is also important and, for this purpose, the adsorbent must be stable during several adsorption-regeneration cycles. In order to analyze the NO adsorption capacity and kinetic behavior of spent samples after regeneration, NO 
adsorption experiments were carried out with samples regenerated at $473 \mathrm{~K}$, and the adsorption-regeneration cycle was repeated for several times. As an example, Figure 4 shows the NO adsorption profile obtained with fresh $\mathrm{CFe} 8$ and with the same during the third NO adsorption step (CFe8r). Similar NO ad sorption capacity is observed during the first and third adsorption steps, even though slightly slower adsorption kinetic occurred in the regenerated sample during the first $30 \mathrm{~min}$ approximately. At higher times, from 30 to 90 minutes, the opposite behaviour occurs and from 90 minutes both curves overlap. The behavior of this sample during the fifth adsorption step is similar to that presented in Figure $4(\mathrm{CFe} 8 \mathrm{r})$. This result is reasonable taking into account that neither fiber gasification nor modification of the iron species occurs during the regeneration steps. It is also important to note that samples present the same NO adsorption capacity after regeneration at $373 \mathrm{~K}$ in stead of $473 \mathrm{~K}$.

\subsubsection{NO adsorption on heat-treated samples.}

Effect of the nature of iron species.

NO adsorption profiles corresponding to samples that were previously thermally treated at $1223 \mathrm{~K}$ are presented in Figure 4, including " $\mathrm{t}$ " after the nomenclature. As observed, all the samples experimented an important NO adsorption capacity increase after the heat-treatment. On the other hand, the NO adsorption capacity of heat-treated samples follows the same trend that the series of fresh samples (compare Figure 1 and 4). In both fresh and treated series, $\mathrm{CFe} 8$ and $\mathrm{CFe} 38$ show the highest and lowest NO adsorption capacity, respectively. It is worth to mention the significant increase in NO 
adsorption capacity of samples $\mathrm{CFe}$ and $\mathrm{CFe} 25$ after the heat-treatment, both reaching comparable activities to that of $\mathrm{CFe} 8 \mathrm{t}$. However, it should be mentioned that the sample CFe8t seems not to be completely saturated after 5 hours and, in consequence, higher NO adsorption is expected. With regard to NO adsorption rate, kinetics on $\mathrm{CFe} 8$ and CFe8t are quite similar but longer time is required for CFe8t saturation due to the improved NO adsorption capacity. Within the treated series of samples, the main differences occurred during the first $30 \mathrm{~min}$ and the higher the fiber burn-off, the faster the NO adsorption rate. Finally, notice the faster kinetics on CFet with regard to CFe.

The important increase of the NO adsorption capacity of samples after the heattreatment has been analyzed in detail. As indicated, the highest the NO adsorption capacity of the sample $\mathrm{CFe} 8$ is expected to be related to the presence of very active iron species and, for this reason, all the samples were heat-treated at $1223 \mathrm{~K}$ in order to reduce the oxidized iron species by carbon. During the heat-treatment, the same iron species are expected to be present in all the samples (metallic $\mathrm{Fe}$ or $\mathrm{Fe} / \mathrm{C}$ species, according to the Fe-C phase diagram [36]). Considering our experimental results, the increase in NO adsorption capacity after the heat-treatment is most probably related to the formation of highly reduced iron species. Kaneko et al. [12-14] reported that NO chemisorption on iron sites strongly enhances NO adsorption on ACF, and a number of studies have been carried out in order to elucidate the effect of the electronic and structural nature of iron species on the NO adsorption capacity. Kaneko et al. proved that the rate-limiting step of $\mathrm{NO}$ chemisorption on $\mathrm{FeOOH}$ is the electronic transference from $\mathrm{FeOOH}$ surface to the electron-acceptor $\mathrm{NO}$ molecule [37]. It has also been suggested that iron-improved $\mathrm{NO}$ adsorption is a consequence of the presence of 
partially reduced iron ions [38]. In the same way, the important increase on NO adsorption capacity observed with $\mathrm{FeOOH}$-containing $\mathrm{ACF}$, after being heat-treated at $773 \mathrm{~K}$, was attributed to the presence of partially reduced $\mathrm{Fe}_{2} \mathrm{O}_{3}$ particles, reaching the highest NO adsorption capacity ever reported $(320 \mathrm{mg} / \mathrm{g})$. Considering the important role of the electrical conductivity of iron species, further studies were focused on the enhancement of conductivity. For instance, it has been reported that $\mathrm{FeOOH}$ doping with $\mathrm{Ti}$ [39] or $\mathrm{Cu}$ [40] enhances significantly the NO adsorption capacity, which was attributed to the improved electrical conductivity of Ti- or Cu-doped iron. Taking into account this background, it seems reasonable to assume that the enhanced NO adsorption capacity of the samples here tested after being treated at $1223 \mathrm{~K}$ could be related to the presence of the reduced iron species formed during the heat-treatment as compared to that of oxidized species present on fresh samples.

We found that the reduction of iron species leads to an increase on the NO uptake that could be related with a better formation of dimer. In order to better understand this interaction, we analysed the results from literature that refer to how NO is bound to iron. In this context, most of the studies about this topic are focused on the myoglobin (a protein that contains an iron center), because of its biological relevance. It is well known that NO is both a $\sigma$-donor and $\pi$-acceptor $[41,42]$. The $\pi$-back bonding depends on the interaction between $d$ valence electrons from the metal and the two degenerated $\pi^{*}$-molecular orbitals from the NO. Hence, depending on the kind of metal and its oxidation state, NO could establish or not a $\pi$-back bonding with the metal and up to different stage. Thus, transition metals late in the d-series, with relatively many electrons, show stabilised d orbitals, the back bonding being negligible. In contrast, 
electron-poor early transition metals show their d orbitals less stabilized and the back bonding is frequently found, which implies the existence of nitrosyl complexes of these metals. It is also known that the increase in the strength of the back bonding strengthens the metal-N bond [42] and related to this it may be noted that $\mathrm{Cr}(\mathrm{NO})_{4}$ is the only known metal nitrosyl complex, whereas the existence of $\mathrm{Fe}(\mathrm{NO})_{4}$ is also claimed at very low temperature, although there is lack of experimental evidence of it. This gives an idea of the weakness of metal-N bond for iron.

According to this, the observation of the bonding of $\mathrm{NO}$ in biological systems is quite interesting. In this context, most of the studies of this topic are focused on the myoglobin (a protein that contains an iron center), by its biological relevance. It has been observed that $\mathrm{NO}$ is bonded to the $\mathrm{Fe}(\mathrm{III})$ center in the oxidized form of myoglobin by back bonding, whereas back bonding is not involved between Fe(II) and NO [43].

Taking into account the above comments, it clear that the more reduced the iron species are found in our samples, the weaker the interaction with the NO is. Hence, on the most oxidized sample, which is the most activated (see Table 1), the NO chemisorption is the strongest from all the studied materials.

When NO is adsorbed in the micropore, the dimerization of NO takes place in these confined spaces in absence of iron species as a consequence of the stronger potential adsorption existing in these micropores, which favours the coupling of molecules [9]. In our materials, both the iron compounds and the NO molecules are 
confined in the narrow microporosity. The presence of iron compounds inside the micropores increases the dimerization and it is reasonable to think that iron compounds can be used as anchoring sites for the NO molecules via bonding. Hence, the formation of dimers between two NO molecules placed in adjacent positions would be more favoured inside the micropore. Obviously, once the dimer has been formed it will be released, so that the dimer would be adsorbed on the carbon surface and the exposed iron surface would be available for new NO molecules. In order this can occur, the dimer-NO bonding should be weak enough. Thus, it seems clear that metallic iron and low-oxidized iron species are preferred, in comparison to more oxidized species.

According to this, even the sample CFe, which shows very poor NO adsorption capacity before the heat-treatment, presents high NO adsorption capacity after the treatment due to the formation of metallic Fe and/or Fe/C compounds. This sample was carbonized at $1173 \mathrm{~K}$ and the further heat-treatment was performed at $1223 \mathrm{~K}$. Important differences were detected between fresh and treated samples, which ind icates that, in addition to the important role of the reduced iron species, other properties of the samples must be also considered, as discussed in the following section.

\section{Effect of the porosity.}

Taking into account that the surface oxygen complexes decomposition occurs during the heat-treatment of samples, it is reasonable to expect that the porous structure could be modified. In order to analyze this phenomenon, the micropore volume of samples after the heat-treatment was determined and the results are compiled in Figure 
5 along with those of the fresh samples. In this figure, the amounts of NO adsorbed have been also included.

As observed in Figure 5, both the narrow $(<0.7 \mathrm{~nm}$; Vc) and total $(<2 \mathrm{~nm}$; Vn) micropore of samples change after the heat-treatment. This indicates that narrow micropores are created and that the existing micropores become wider during the heattreatment. However, the micropore volume development is different in each sample: micropore volume development on $\mathrm{CFet}$ and $\mathrm{CFe} 25 \mathrm{t}$ is the highest, $\mathrm{CFe} 8 \mathrm{t}$ micropore volume slightly increases, and micropore volume of CFe38t even decreases after the treatment. Note that the micropores of CFet and CFe25t do not become wider while this effect strongly affects CFe38t.

Considering the NO adsorption capacity of the different samples, it can be concluded that both, reduced iron species and a suitable microporosity are required for high NO uptake. This is clearly deduced by comparison of samples CFe and CFe38t. Both samples contain the same iron species but different pore size. CFe is characterized by a very narrow micropore size, and as discussed in section 3.1.2., the $\mathrm{N}_{2} \mathrm{O}_{2}$ dimer formation being hindered within this type of narrow microporosity. On the contrary, CFe38t presents the widest micropores (supermicropores) among the heat-treated samples. The ratio between pore diameter and molecular size of the adsorbed gas plays a major role in the adsorption potential for gas adsorption in micropores [44]. Therefore, for a pore diameter/molecular size ratio near 1 , the adsorption potential is very high but the adsorption capacity is very low because only a single layer of gas can be adsorbed. On the contrary, for pore diameter/molecular size ratios above 2, the adsorption 
potential is low but there is more volume available for gas adsorption. Therefore, the low NO adsorption capacity of $\mathrm{CFe} 38 \mathrm{t}$ must be attributed to presence of a high content of sup ermicropores.

\subsection{Comparison of samples prepared in the current study with data in the literature.}

Well-defined microporosity with proper pore size distribution is a key requirement for $\mathrm{NO}$ adsorption on $\mathrm{Fe}-\mathrm{ACF}$, whatever the preparation procedure is. In Table 2, the NO adsorption capacity of different ACF samples reported in the open literature $[9,32]$ is compiled, which were determined in a highly concentrated NO gas stream $(80 \mathrm{kPa})$. In Table 2 , the micropore volumes of these ACF are also included along with results obtained in the current study $(5 \mathrm{kPa})$. Comparison of data is difficult because some of the samples included in Table 2 do not contain iron (P10, P15, and ACF-ox), and the nature of the iron species on the remaining samples is not the same. However, despite these differences, data in Table 2 indicate that NO retention on samples with low $\mathrm{N}_{2}$ volume is higher. For instance, P10 does not contain iron but presents a high NO adsorption capacity. Sample a-ox-acf-h was prepared by heattreatment at $673 \mathrm{~K}$ of a-ox-acf [32], and an interesting relationship between the increase of NO adsorption capacity after the treatment and Vn decrease is observed. However, the sample CFe38t shows the opposite behavior, and the $\mathrm{Vn}$ of this sample increases and NO adsorption capacity also does (see Figure 5). Vc characterization also provides useful information. As deduced from data in Table 2, little difference between Vc and $\mathrm{Vn}$ is required for suitable NO capture. Kaneko et al. [8] reported that the optimum pore 
diameter for $\mathrm{NO}$ adsorption is $0.6-1.1 \mathrm{~nm}$, and the difference between $\mathrm{Vc}$ and $\mathrm{Vn}$ provides information about supermicroporosity $(>1.4 \mathrm{~nm})$ on samples.

It has been reported that oxygen-complexes in the fiber surface also play an important role in NO adsorption [45]. However, results obtained in the current study with heat-treated samples that do not contain this type of groups suggest that surface oxygen-carbon complexes do not affect NO adsorption significantly.

Finally, it is important to underline the usefulness of the Fe-ACF preparation procedure developed in the current study, which is more convenient than the conventional impregnation method. Higher metal dispersion is obtained, which is related to the high $\mathrm{NO}$ adsorption capacity, despite the low iron content $(0.1 \mathrm{wt} . \%)$. It is observed that the NO adsorption capacity of sample CFet is comparable to those of the best samples. This is even more important taking into account the preparation procedure of the different samples:

- In the current study, the samples were easily prepared: A petroleum pitch suitable for $\mathrm{CF}$ manufacturing was mixed with a very small amount of $\mathrm{FeCl}_{3}(0.1 \mathrm{wt} . \%)$, not modifying the pitch features, and Fe-CF is obtained after spinning.

- The remaining samples were prepared following a complex procedure that involves five steps, at least: i) CF preparation, ii) CF activation obtaining ACF, iii) ACF oxidation, iv) ACF impregnation and washing, and v) heat-treatment.

Therefore, the preparation procedure used in the current study is cheaper (fewer steps are required and a higher yield is achieved), easier, and more efficient. 


\section{Conclusions.}

In this study, iron-containing ACF have been prepared by a novel method consisting of mixing an iron precursor with raw pitch before carbon fiber formation, and further $\mathrm{CO}_{2}$ activation of the Fe-CF. This is a promising method because it is easier than reported methods and produces finely dispersed metallic particles. Iron species are accessible to gas and activ ate $\mathrm{NO}$, improving adsorption into the fibers microporosity.

NO adsorption on ACF takes place in two steps: i) NO chemisorption and dimer formation and ii) dimer diffusion throughout the micropores. The adsorption rate of NO on Fe-ACF is, in general, controlled by the first step, and for this reason, iron species improve adsorption. However, the second step is slowest in samples with narrow micropores due to the hinder dimer diffusion.

NO adsorbed can be further desorbed by heating the spent adsorbents at quite low temperature $(500 \mathrm{~K})$, and fresh and regenerated adsorbents show similar NO adsorption capacity.

Metallic iron and/or partially reduced iron are the most active iron species, as deduced from NO adsorption experiments on heat-treated samples. In addition, an appropriate pore size is required from a kinetic point of view. Most suitable samples prepared were those with medium activation degree, because they combine microporosity of pore size larger than $0.7 \mathrm{~nm}$ (narrow microporosity) and a content of partially reduced iron species. 


\section{Acknowledgments}

The authors thank the Spanish Government (project CTQ2005-01358) for financial support. 


\section{References}

[1] J.B. Donnet, T.K. Wang, J.C.M. Peng, S. Rebouilla, Carbon Fibers, 3rd edition, Dekker, New York, 1998..

[2] T.J Mays,. Activated carbon fibers. In: Burchell TD, editor. Carbon materials for advanced technologies, Oxford: Pergamon, 1999; p 95-118

[3] M. Suzuki, M. Carbon 32 (1994) 577.

[4] R.N. Mcnair, G.N. Arons. Sorptive textile systems containing activated carbon fibers. In: Cheremisinoff PN, Ellerbusch F, editors. Carbon Adsorption Handbook, Ann Arbor, Mich.; Ann Arbor Science: 819-60.

[5] S.N. Ahmed, R. Baldwin, F. Derbyshire, B. McEnaney, J. Stencel, Fuel 72 (1993) 287.

[6] H. Teng, E.M. Suuberg, J.M. Calo, Energy and Fuels 6 (1992) 398.

[7] M.J. Illán-Gómez, A. Linares-Solano, L.R.Radovic, C. Salinas-Martínez de Lecea, Energy and Fuels 10 (1996) 158.

[8] K. Kan eko, N. Fukuzaki, K. Kakei, T. Suzuki, S. Ozeki, Langmuir 5 (1989) 960.

[9] K. Kan eko, Y. Nakahigahi, K. Nagata, Carbon 26(1988) 327.

[10] C.M. Yang, K.Kaneko, J. Colloid Interface Science 255 (2002) 236.

[11] K. Otto, M. Shelef, J. Catal. 18 (1970) 184.

[12] K. Kan eko, Langmuir 3 (1987) 357.

[13] K. Kan eko, N. Shindo, Carbon 27 (1989) 815.

[14] Z.M.Wang, T.Suzuki, N. Uekawa, K Asakura, K. Kaneko, J. Phys. Chem. 96 (1992) 10917. 
[15] J. Alcañiz-Monge, M.A. Lillo-Ródenas, A. Bueno-López, M. Illán-Gómez, Microporous \& Mesoporous Materials 100 (2007) 202.

[16] J. Alcañiz-Monge, D. Cazorla-Amorós, A, Linares-Solano, S.Yoshida, A. Oya, Carbon 32 (1994) 1277.

[17] F. Rodríguez-Reinoso, A. Linares-Solano. Microporous structure of activated carbons as revealed by adsorption methods. In: P.A. Thrower, editor. Chemistry and Physics of Carbon, vol 21, Dekker, New York, 1988, 1.

[18] H. Teng, E.M. Suuberg, J. Phys. Chem. 97 (1993) 478.

[19] D.M. Ruthven, Principles of adsorption and adsorption processes. Wiley: New York, 1984.

[20] M.J.D.Low, Chem. Rev. 60 (1960) 267.

[21] G.F. Cerofolini, Adsorption Kinetics on real Surfaces. In: A. Darbrowski, V.A. Tertykh, editors. Adsorption on New and Modified inorganic adsorbents, Elsevier:Amsterdan, 1995; p 435-65.

[22] A.S. Porter, F.C. Tompkins, Proc. Roy. Soc. (London) A 217 (1953) 529.

[23] C.E. Brown, P.G. Hall, Trans. Faraday Soc. 67 (1971) 3558.

[24] K. Kaneko, C. Ishii, Colloids and surfaces 67 (1992) 203.

[25] M. Sato, T. Sukegawa, T. Suzuki, K. Kaneko, J. Phys. Chem. B. 101 (1997) 1845.

[26] H. Birnholtz, A. Nir, N. Lotan, C. Aharoni, Canadian Journal of Chemical Engineering 62 (1984) 233.

[27] I. Mochida, M. Ogaki, H. Fujitsu, Y. Komatsubara, Fuel 64 (1985) 1054.

[28] H. Teng, E.M. Suuberg, Ind. Eng. Chem. Res. 32 (1993) 416.

[29] J. Imai, T. Suzuki, K. Kaneko, Catal. Lett. 20 (1993) 133. 
[30] K. Kan eko, A. Kobayashi, T. Suzuki, S. Ozeki, K. Kakei, N. Kosugi, H. Kuroda, J. Chem. Soc. Faraday Trans. I. 84 (1988) 1795.

[31] Y. Nishi, T. Suzuki, K. Kaneko, J. Phys. Chem. B. 101 (1997) 1938.

[32] K. Kaneko, S. Ozeki, K. Inouye, Colloid \& Polymer Sci. 265 (1987) 1018.

[33] I. Aarna, E.M. Suuberg, Fuel 76 (1997) 475.

[34] A. Newkirk, Anal. Chem. 32 (1960) 1558.

[35] M. J. Illán-Gómez, A. Linares-Solano, C. Salinas-Martínez de Lecea, Energy and Fuels 9 (1995) 976.

[36] J.D. Fast, Interaction of metals and gases. Academic Press: New York, 1965, 6170.

[37] K. Kan eko, K.Inouye, Adsorption Science \& Technology 3 (1986) 11.

[38] J. Imai, M. Souma, S. Ozeki, T. Suzuki, K. Kaneko, J. Phys. Chem. 95 (1991) 9955.

[39] Z.M. Wang, T. Suzuki, N. Uekawa, K. Asakura, K. Kaneko, J. Phys. Chem. 96 (1992) 10917 .

[40] Z.M. Wang, N. Shindo, Y. Otake, K. Kaneko, Carbon 32 (1994) 515.

[41] H.W. Roesky, K.K. Pandey, Adv. Inorg. Chem. Radiochem. 26 (1983) 337.

[42] F.A. Cotton, G. Wilkinson, C.A. Murillo, M. Bochmann, Advanced Inorganic Chemistry, $6^{\text {a }}$ ed. John Wiley \& Sons, Inc.: New York, 1999, 641.

[43] A.M. Rich, R.S. Armstrong, P.J. Ellis, P.A. Lay, J. Am. Chem. Soc. 120 (1998) 10827.

[44] D.H. Young, A.O. Crowell, Physical Adsorption of Gases; Butterworth: London, 1962

[45] B.M. Hoffman, N.J. Nelson, J. Chem. Phys. 50 (1969) 2598. 
Table 1. Fe-ACF pore volumes as determined by $\mathrm{N}_{2}(\mathrm{Vn})$ and $\mathrm{CO}_{2}(\mathrm{Vc})$ adsorption and iron species contained in these fibers

\begin{tabular}{|l|l|l|l|}
\hline & Vn $\left(\mathbf{c m}^{3} / \mathbf{g}\right)$ & $\mathbf{V c ~}\left(\mathbf{c ~ m}^{3} / \mathbf{g}\right)$ & Iron species (XRD) \\
\hline $\mathbf{C F e}$ & 0.13 & 0.15 & $\mathrm{Fe} / \mathrm{C}, \mathrm{Fe}_{3} \mathrm{C}, \mathrm{Fe}$ \\
$\mathbf{C F e 8}$ & 0.26 & 0.24 & $\mathrm{Fe}, \mathrm{FeO}$ \\
$\mathbf{C F e 2 5}$ & 0.36 & 0.31 & $\mathrm{FeO}, \mathrm{Fe}_{3} \mathrm{O}_{4}$ \\
$\mathbf{C F e 3 8}$ & 0.43 & 0.20 & $\mathrm{Fe}_{3} \mathrm{O}_{4}, \mathrm{Fe}_{2} \mathrm{O}_{3}$ \\
$\mathbf{C F e 5 0}$ & 0.46 & 0.25 & $\mathrm{Fe}_{2} \mathrm{O}_{3}$ \\
\\
Vn: pore volume determined by $\mathbf{N}_{2}$ adsorption at $77 \mathrm{~K} ;$ Vc: pore volume determined by $\mathbf{C O}_{2}$ \\
adsorption at 273K
\end{tabular}


Table 2. Pore volumes and NO adsorption capacity of different ACF samples reported in the literature.

\begin{tabular}{|c|c|c|c|}
\hline & $\mathrm{Vn}\left(\mathrm{cm}^{3} / \mathrm{g}\right)$ & $\mathrm{Vc}\left(\mathrm{cm}^{3} / \mathrm{g}\right)$ & $\mathrm{NO}(\mathrm{mg} / \mathrm{g})$ \\
\hline $\mathbf{P 1 0}[9]^{\mathrm{a}}$ & 0.45 & - & 72 \\
\hline$P 15[9]^{a}$ & 0.60 & - & 53 \\
\hline $\operatorname{ACF}-0 x[32]^{a}$ & 0.62 & 0.26 & 50 \\
\hline a-ox-acf $[32]^{a}$ & 0.63 & 0.28 & 90 \\
\hline a-ox-acf-h $[32]^{a}$ & 0.37 & 0.20 & 162 \\
\hline CFe8t ${ }^{b}$ & 0.28 & 0.25 & 115 \\
\hline $\mathrm{CFe} 38 \mathrm{t}^{\mathrm{b}}$ & 0.52 & 0.19 & 88 \\
\hline
\end{tabular}

$\mathrm{NO}$ adsorption at $300 \mathrm{~K}$ at: ${ }^{\mathrm{a}} 80 \mathrm{kPa} ;{ }^{\mathrm{b}} 5 \mathrm{kPa}$ 


\section{Figure Captions}

Figure 1.- NO ad sorption curves obtained at $300 \mathrm{~K}$ for Fe-ACF.

Figures 2 a-c. Kinetic curves obtained by application of analytical equation of Fick's Law to NO adsorption data: (a) at short times and (b) long times. (c) Kinetic curves obtained by application of Elovich equation to NO adsorption data.

Figure 3. Thermogravimetry desorption curves during heat-treatment at $1273 \mathrm{~K}$ for Fe-ACF samples after NO adsorption for 4 hours.

Figure 4. NO adsorption curves obtained at $300 \mathrm{~K}$ for: untreated CFe8, regenerated CFe8, CFe8r, and all Fe-ACF heat-treated at $1223 \mathrm{~K}$ (subscript t).

Figure 5. Micropore volumes (bars) and the amounts of adsorbed NO (triangles) for original Fe-ACF and heat-treated Fe-ACF at $1223 \mathrm{~K}$ 


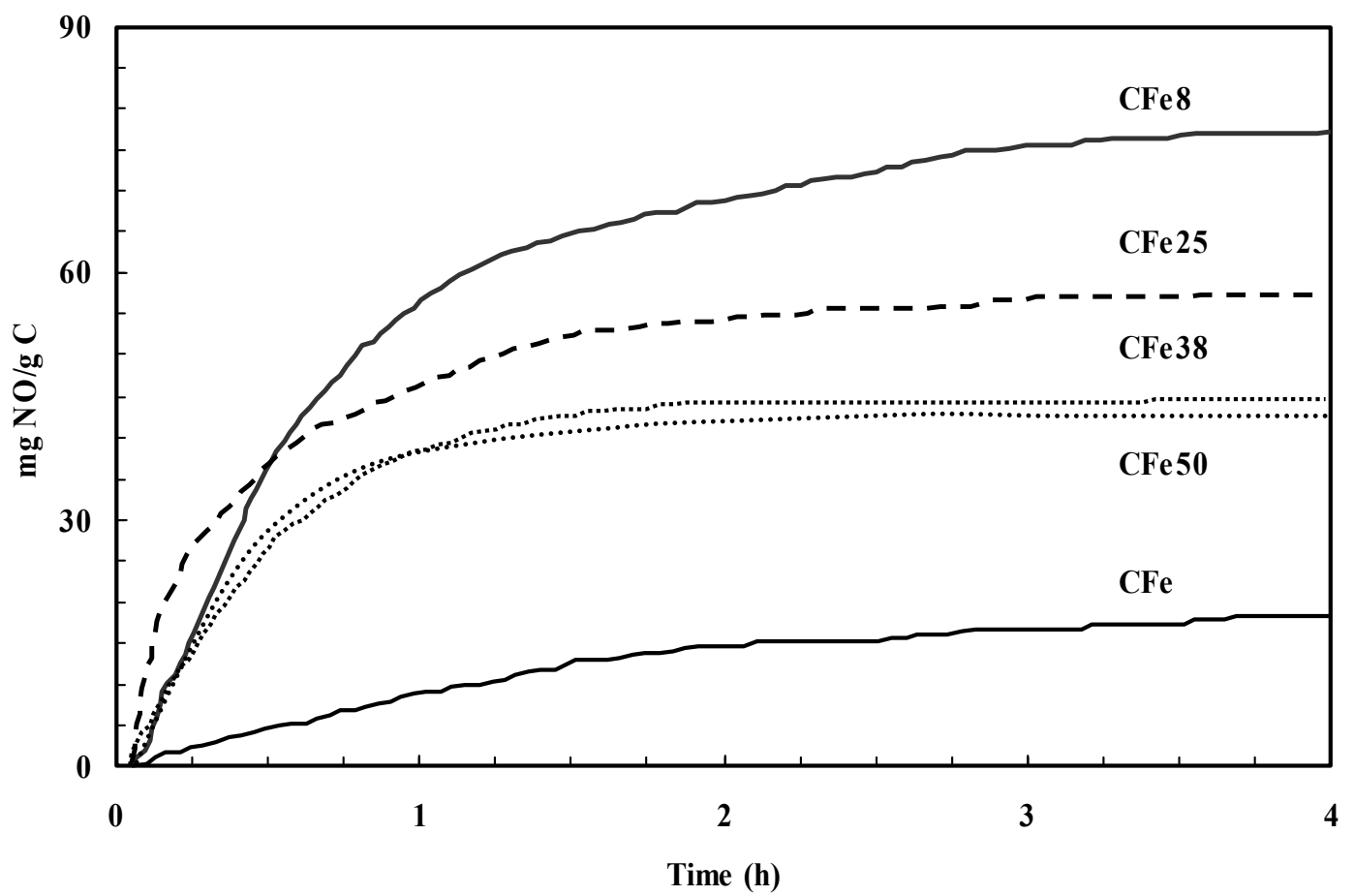

Figure 1. NO adsorption curves obtained at $300 \mathrm{~K}$ for Fe-ACF. 
(a)

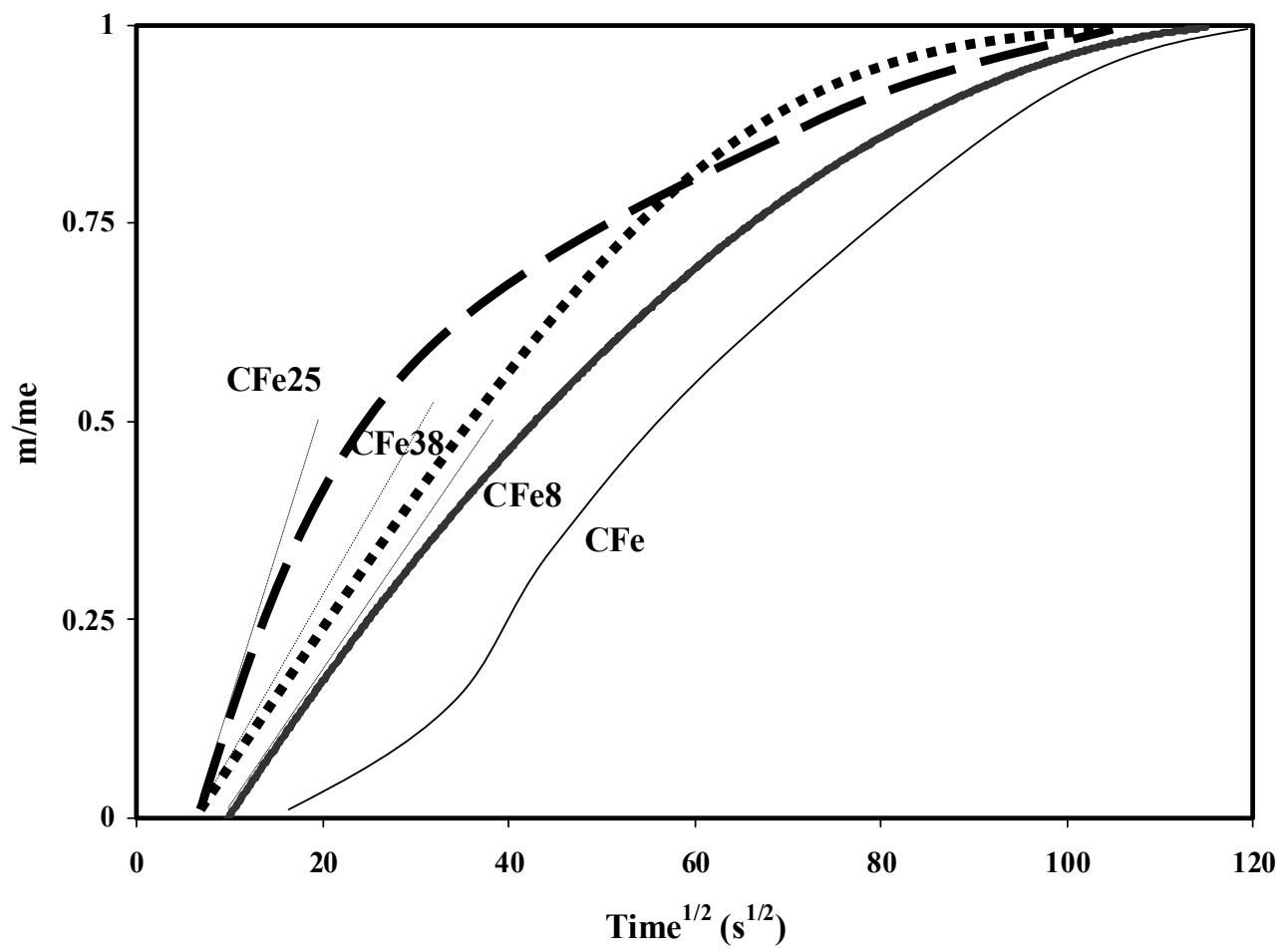

(b)

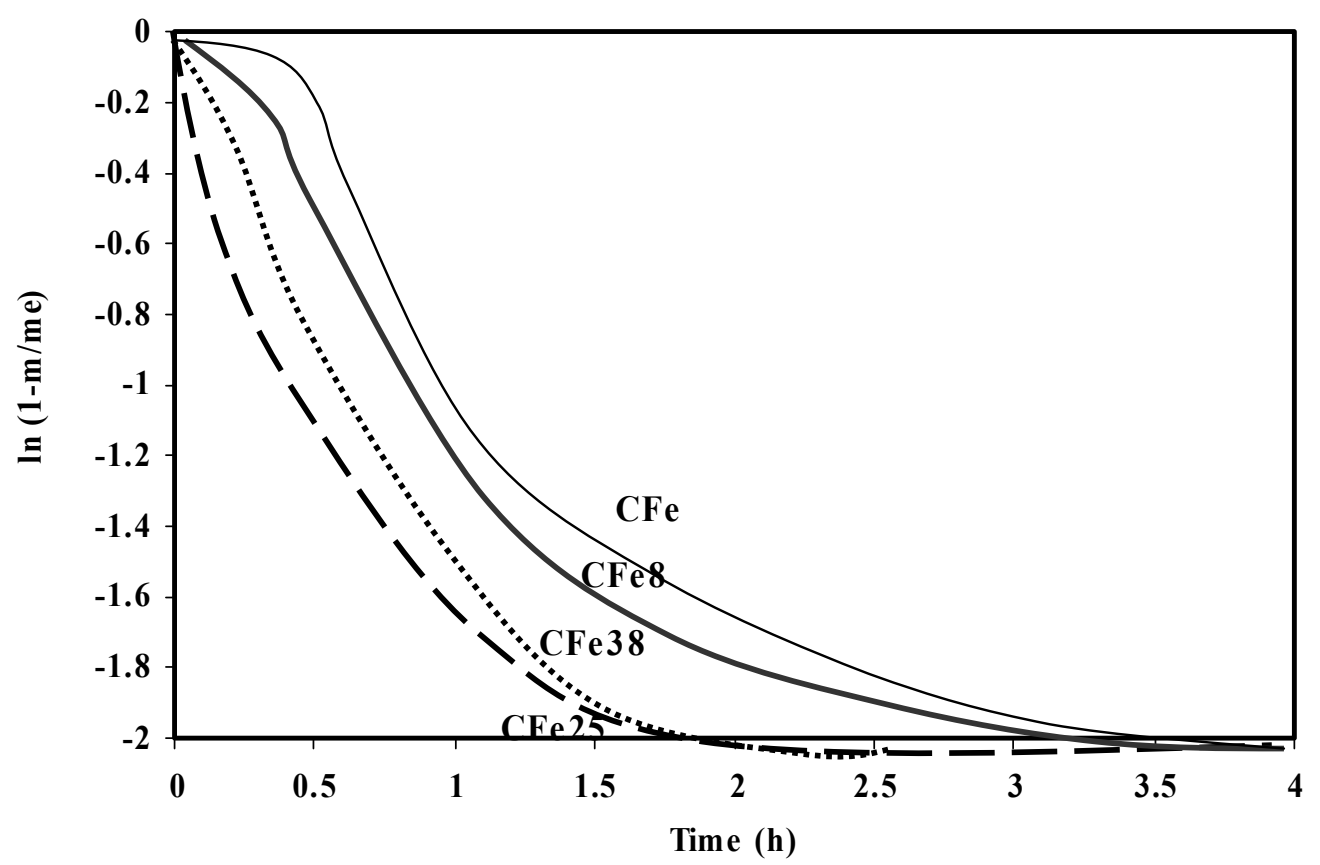


(c)

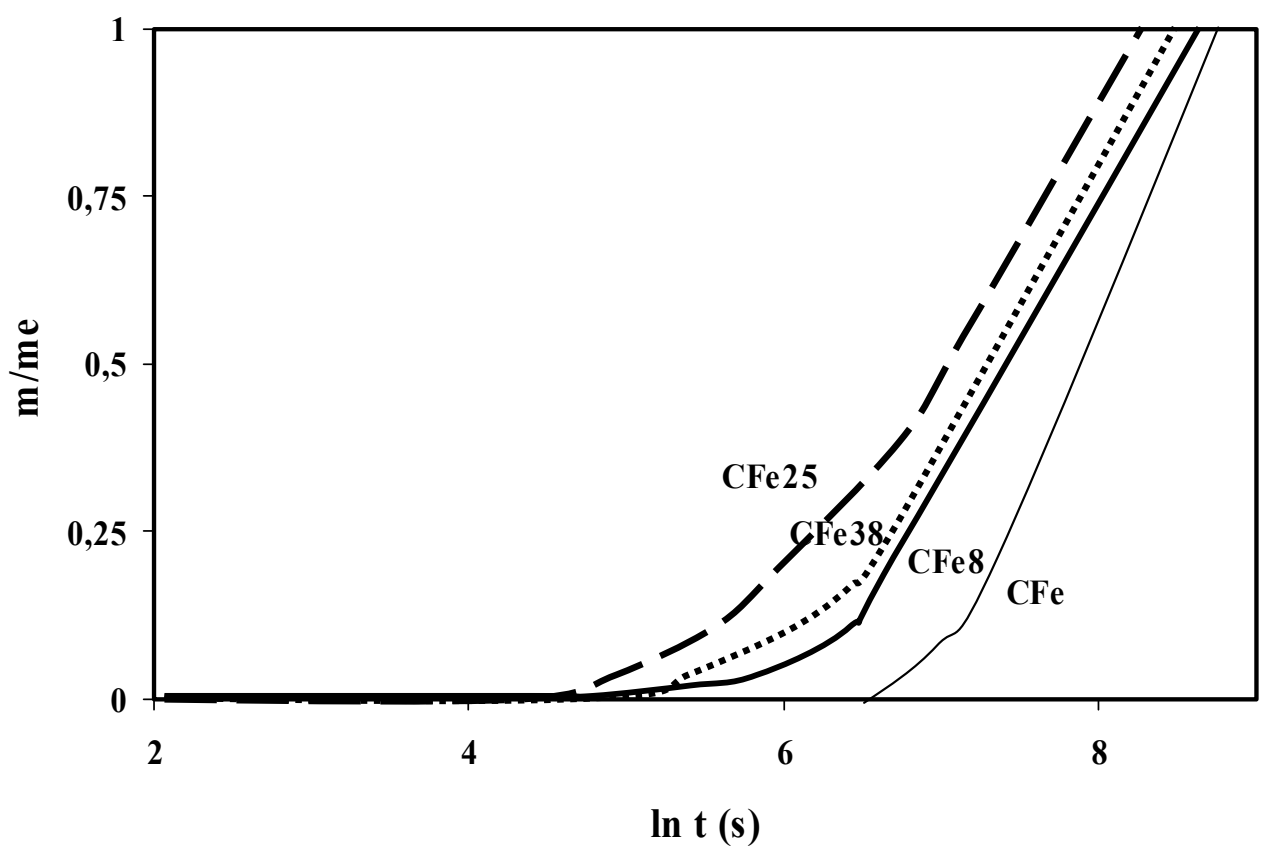

Figures 2 a-c. Kinetic curves obtained by application of analytical equation Fick's Law to NO adsorption data: (a) at short times and (b) long times. (c) Kinetic curves obtained by application of Elovich equation to NO adsorption data. 


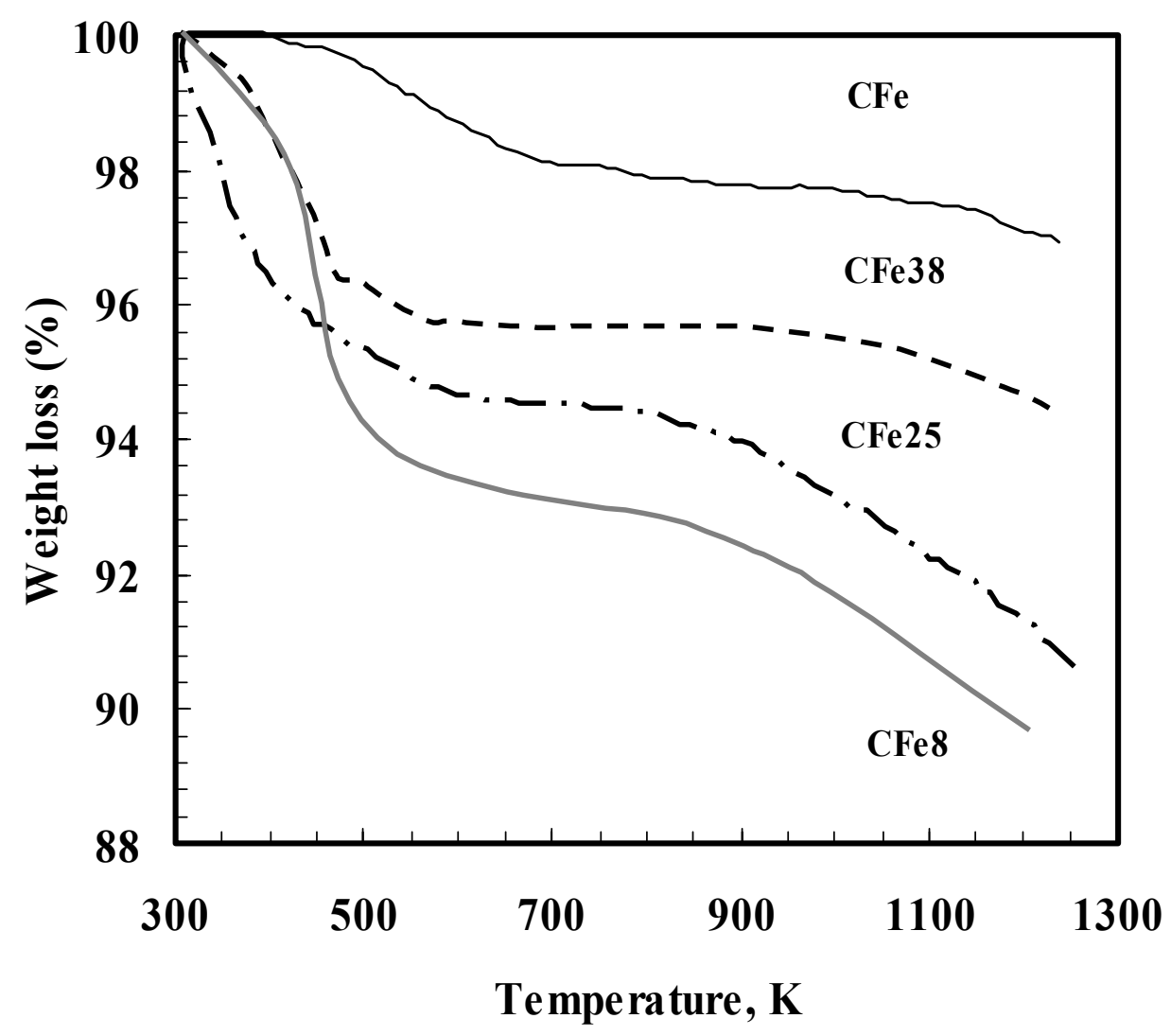

Figure 3. Thermogravimetry desorption curves during heat-treatment at $1273 \mathrm{~K}$ for Fe-ACF samples after NO adsorption for 4 hours. 


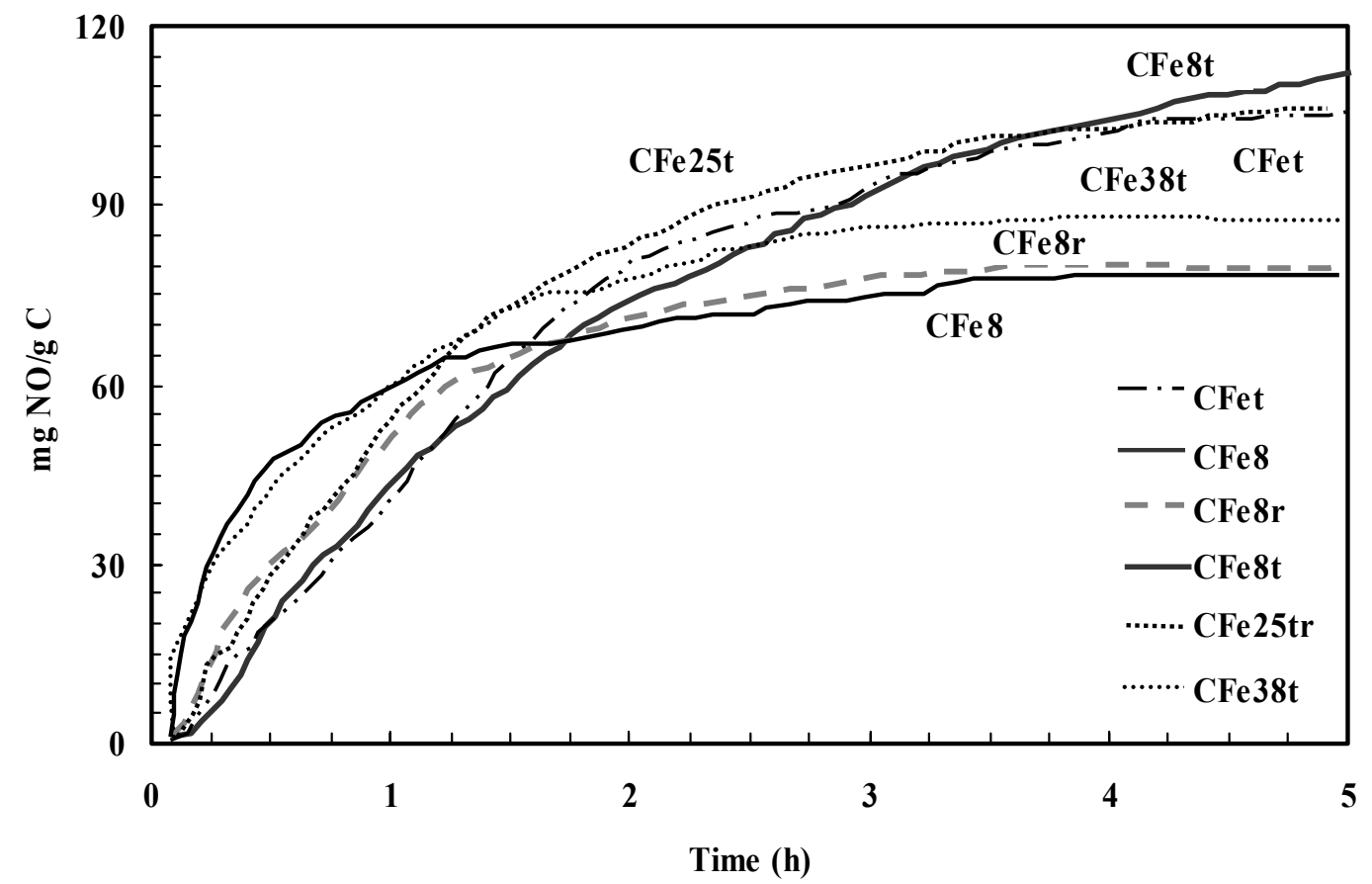

Figure 4. NO adsorption curves obtained at $300 \mathrm{~K}$ for: untreated CFe8, regenerated CFe8, CFe8r, and all Fe-ACF heat-treated at $1223 \mathrm{~K}$ (subscript t). 


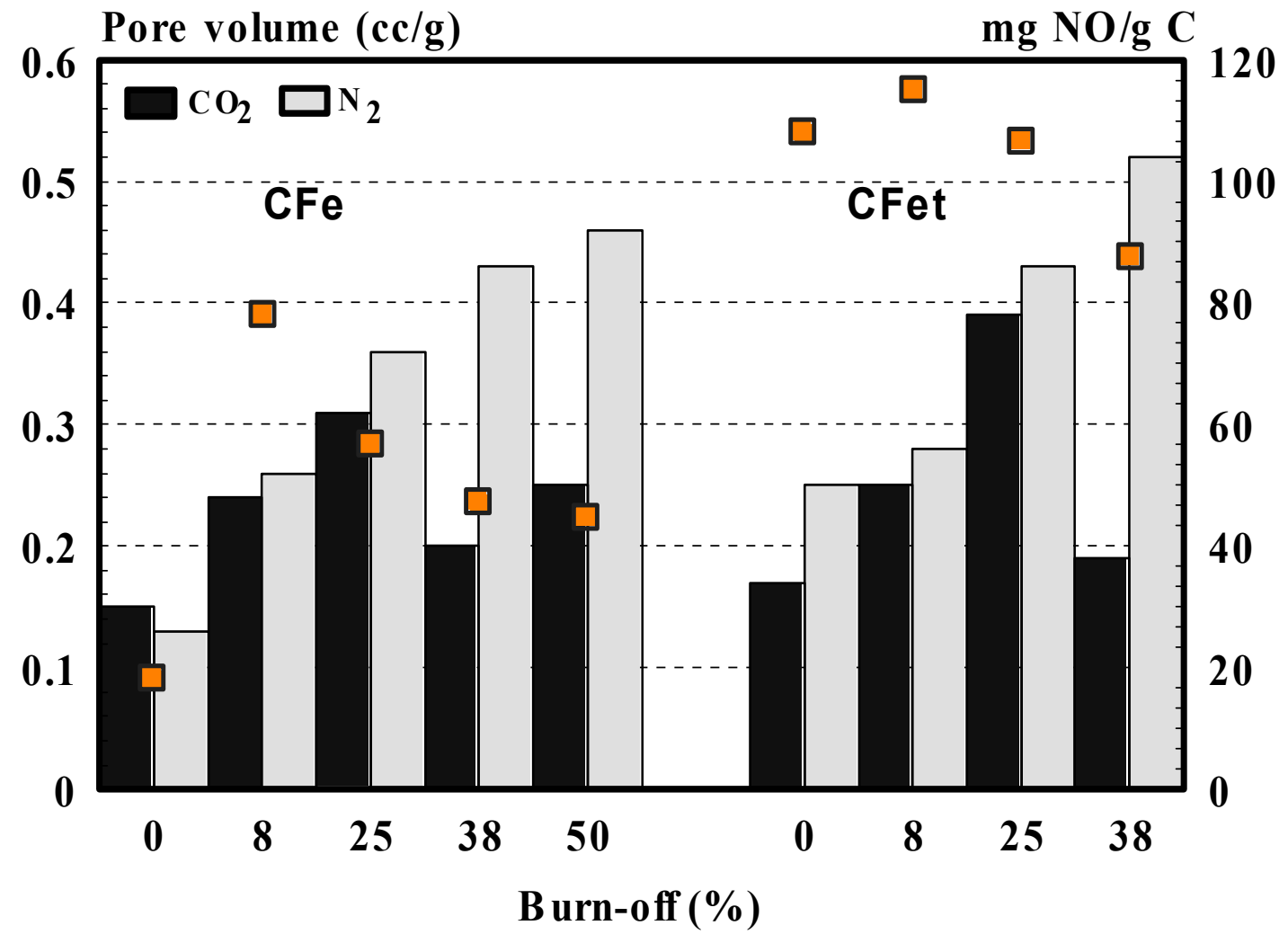

Figure 5. Micropore volumes (bars) and the amounts of adsorbed NO (triangles) for original Fe-ACF and heat-treated Fe-ACF at $1223 \mathrm{~K}$ 